\title{
照 Springer
}

http://www.springer.com/0-387-23641-4

Neuroendocrine Correlates of SleepMakefulness

(Eds.)D.P. Cardinali; S.R. Pandi-Perumal 2006, XXII, 627 p. 103 illus., Hardcover ISEN: 0-387-23641-4 


\title{
Basic Mechanisms of Circadian Rhythms and their Relation to the Sleep/Wake Cycle
}

\author{
Martha U. Gillette and Sabra M. Abbott
}

\section{The Temporal Spectrum of Life}

Organisms exhibit cyclic variations in a variety of essential functions, including the sleep-wake cycle, hormonal regulation, and reproduction. A primary environmental signal regulating these functions is the daily alternation of darkness and light exerted by the rotation of the earth. Superimposed upon the daily light-dark cycle is a seasonal influence that modifies the relative durations of day and night over the course of a year. These environmental changes make it necessary for organisms to be able to modify their behavior so that they are active during times when the opportunity to acquire nutritional resources exceeds the risk of predation, and resting during times when the need for vigilance is minimized. Be they day-active or night-active, all organisms need a means of keeping time in a 24-hour world and adjusting to changes in day length or transition times that may occur.

As any observer of the natural world knows, an organism's active behaviors generally occur in bouts that recur at a predictable phase of the cycle of day and night. This cyclic organization of behavior is expressed in the patterning of wheel-running activity of rodent models (Figure 1). Figure 1A depicts the activity of an animal under conditions where the lights are on for 12 hours and off for 12 hours. This phenomenon is called entrainment, in which animals express activity with a fixed phase relationship to environmental conditions.

At first, one might assume that these circadian rhythms are simply a reflection of the external day-night cycle. However, when all exogenous timing cues are removed, these rhythms persist. Every organism expresses an endogenous rhythm that varies slightly from 24 hours, making it circadian, or 'about a day.' Unperturbed, this circadian rhythm persists, as can be seen in Figure 1B. Due to the slight deviation of the period of the rhythm from 24 hours under these constant environmental conditions, the onset of activity drifts from its original position by a small but constant amount each day. In an aperiodic environment, the animal's activity eventually would become completely out of phase with the environment and then pass back into phase, proceeding at a regular interval with each circadian cycle. This 

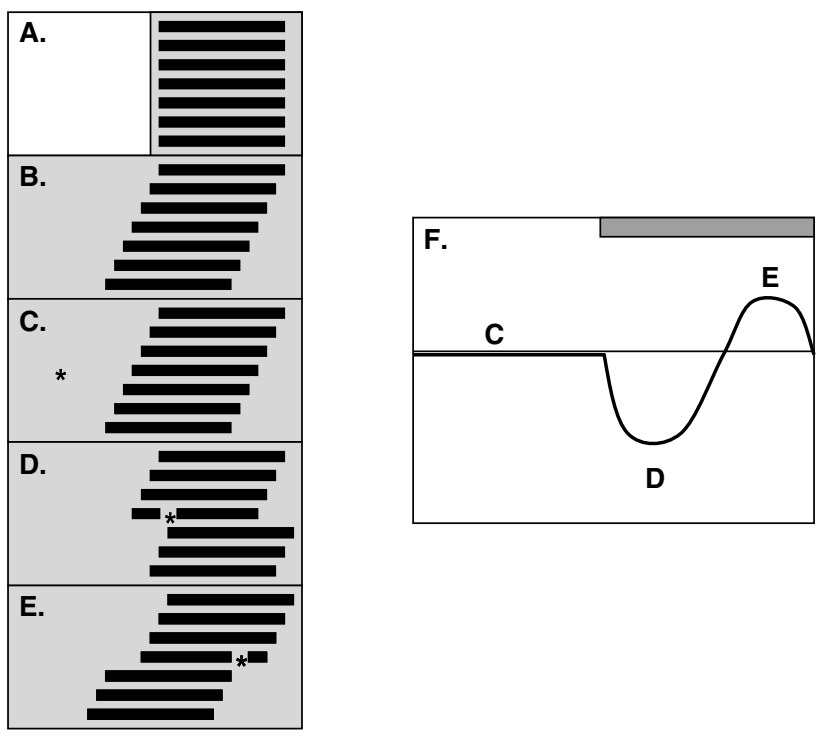

FIGURE 1. Organization of circadian rhythms of locomotor activity and its regulation by light. Panels A-E are schematic actograms from a nocturnal rodent. Dark bars in all figures indicate time when the animal is active, and grey areas indicate time when the animal is in darkness. Black bars indicate running-wheel activity. A. depicts an animal that has entrained to a light-dark cycle. Activity occurs with a regular 24-h cycle, and primarily during darkness. B. represents the same animal placed under constant conditions, showing a free-running period (tau) of less than $24 \mathrm{~h}$. Activity begins slightly earlier each day. C. represents the effect on phase of activity onset in an animal presented with a light pulse $(*)$ during the subjective daytime. No change is observed in the onset of activity on the following day. D. shows the response to a light pulse $\left(^{*}\right)$ during the subjective early evening. Activity onset is delayed on the following cycles. E. shows the response to a light pulse $(*)$ during the subjective late evening. The onset of activity occurs earlier than predicted on the following cycles. F. is a phase response curve (PRC) for the response to light presented at various circadian phases for animals in constant darkness. The grey bar represents subjective night, and deflection above or below the line represents advances or delays, respectively. Times of treatment for the representative actograms (left panel) are represented by the corresponding letters on the graph.

demonstrates that the persistence of rhythmicity is not simply an after-effect of the previous environmental conditions, but rather that an endogenous rhythm is present.

This persistent endogenous rhythm is the expression of an internal circadian clock. The daily period of this biological clock differs from 24 hours by a small, but measurable amount. The circadian clock keeps time without 
external timing signals and, in turn, times circadian rhythms in brain and body functions.

In addition to timing behavioral rhythms, the circadian clock also can influence the levels of a variety of hormones, and many of these can be used as markers of the circadian clock. For example, plasma corticosterone shows a peak shortly before waking, ${ }^{1}$ while plasma melatonin normally peaks shortly after sleep onset and appears to play a role in regulation of the sleepwake cycle. ${ }^{2}$ Circadian rhythms can also be observed in glucose metabolism; these are independent of daily food intake, although feeding and drinking behaviors are themselves circadian rhythms. ${ }^{3}$

Regardless of the marker of circadian phase that is being examined, however, one unique property of circadian clocks is their ability to be reset by environmental signals. The most prominent of these signals is light. When an animal in constant conditions is presented with a brief $(\sim 15 \mathrm{~min})$ pulse of light, the response of the animal depends upon the time of light exposure (Figure 1C-E). During the subjective daytime, the inactive period for this animal, light has little effect. However, light exposure during subjective early evening, the beginning of the active period, delays the onset of activity on the following cycle, and subsequent rhythms continue from this new phase. On the other hand, light exposure during the subjective late night will advance the onset of activity on the following day. A plot called a phase response curve (PRC) can be constructed demonstrating the observed circadian response following treatments at various phases of the day/night cycle, as seen in Figure 1F.

When an animal is housed in conditions with exogenous time cues, such as a light-dark cycle, time is measured as zeitgeber time (ZT), because of the “time-giving' quality of light in the environment. ZT 0 is designated as the time of light onset. If an animal is housed in conditions without exogenous time cues and is relying on the endogenous clock for timing, we discuss time of day in terms of circadian time (CT), where CT 12 in nocturnal animals is designated as the time of onset of activity. The endogenous period of the animal is referred to as $\tan (\tau)$. CT differs from ZT by a factor of $24 / \tau$.

\subsection{The Central Clock in the Suprachiasmatic Nucleus}

Now that we have an idea of the many rhythms that are endogenously regulated and the fact that they can be controlled by environmental signals such as light, the question arises as to what might be controlling these rhythms. In mammals, circadian rhythms are regulated by a paired brain nucleus located at the base of the hypothalamus, directly above the optic chiasm, hence the name - the suprachiasmatic nucleus (SCN). Multiple experiments have demonstrated the role of the $\mathrm{SCN}$ as a central pacemaker for circadian rhythms. Lesioning studies found that selectively damaging the SCN disrupts rhythmicity in corticosterone levels, drinking activity, and wheel-running 
behavior. ${ }^{4,5}$ This provided the initial evidence that the central pacemaker for the mammalian circadian clock lay within the SCN.

In later work, it was found that transplanting fetal SCN tissue into the third ventricle of animals in which the SCN had been lesioned could restore rhythmicity. ${ }^{6}$ Furthermore, if fetal SCN tissue from a wild-type hamster was implanted into a hamster with a shortened free-running period, the new freerunning period resembled that of the SCN donor rather than the host animal. This evidence suggested that not only was the SCN necessary for generating rhythms, but also that this rhythmicity was an intrinsic property of the SCN cells, which could drive the rhythms of the entire animal. ${ }^{7}$

In the rat, each SCN measures approximately $300 \mu \mathrm{m}$ medial to lateral, $300 \mu \mathrm{m}$ dorsal to ventral, and spans approximately $700 \mu \mathrm{m}$ from rostral to caudal end. One SCN contains a total of approximately 8,000-10,000 cells, occupying a volume of approximately $0.036 \mathrm{~mm}^{3}{ }^{8}$ Based on peptide localization, it is common to divide the rat SCN into a ventrolateral or 'core' region, and a dorsomedial or 'shell' region. The core neurons are smaller $\left(\sim 30 \mu \mathrm{m}^{2}\right)$ and contain vasoactive intestinal peptide (VIP), somatostatin and gastrin-releasing peptide (GRP) colocalized with $\gamma$-amino butyric acid (GABA), while the shell neurons are larger $\left(\sim 45 \mu \mathrm{m}^{2}\right)$ and contain arginine vasopressin (AVP). ${ }^{9}$ There are topographic connections between the contralateral shells and the contralateral cores, as well as bidirectional innervation between the core and shell within each nucleus, but the core of one nucleus does not communicate directly with the shell of the contralateral nucleus. ${ }^{8}$

\subsubsection{Outputs and the Circadian Timing System}

The SCN exerts its influence on the rest of the body primarily by sending projections to the rest of the hypothalamus. Neurons from the core region project to the lateral region of the subparaventricular zone (sPVHz), the perisuprachiasmatic area (PSCN) and the ventral tuberal area (VTU), all within the hypothalamus. The destinations of shell region projections include the medial preoptic area (MPOA), medial sPVHz and the dorsal medial hypothalamus (DMH), also all within the hypothalamus. ${ }^{9}$ The DMH projections are particularly interesting, as many of these neurons appear to be projecting to neurons containing hypocretin/orexin, a peptide well known for its role in arousal. ${ }^{10}$ The SCN also contains a minor set of efferents to the ventrolateral preoptic nucleus (VLPO), a region that produces prolonged reduction in sleep duration and amplitude when lesioned. ${ }^{11}$ In addition, the SCN contains projections to the paraventricular nucleus (PVN) of the thalamus, as well as the intergeniculate leaflet (IGL). The targets of these efferents consist of endocrine neurons, autonomic neurons or intermediate neurons that potentially serve to integrate a number of hypothalamic signals. ${ }^{12}$ Overall, the SCN appears to be uniquely situated within a network that allows it to interact closely with the regions controlling the sleep and arousal states of the animal. 
One of the major outputs of the SCN appears to be an inhibitory signal for activity. Two recently discovered candidate factors for communicating such signals include transforming growth factor- $\alpha$ (TGF- $\alpha$ ) and prokineticin 2 (PK2). Under normal conditions, TGF- $\alpha$ is expressed rhythmically in the $\mathrm{SCN}$, and when infused continuously into the cerebral ventricles, it inhibits locomotor activity. Conversely, mice lacking the epidermal growth factor (EGF) receptor, making them unable to respond to TGF- $\alpha$, show an excessive amount of daytime activity. ${ }^{13}$ PK2 is also expressed rhythmically, and can inhibit locomotor activity when infused continuously. ${ }^{14}$ This suggests a role for SCN outputs in promoting an inactive state that would be permissive for sleep.

\subsubsection{Inputs and Gating by the Circadian Clock}

The circadian clock in the SCN also is capable of being reset by changes in behavioral or environmental state. The changes are conveyed to the SCN by projections from a variety of different brain regions.

One of the most well-studied inputs to the SCN comes from subpopulation of retinal ganglion cells that form the retinohypothalamic tract (RHT). Lesions of the SCN disrupt the development of these neurons, ${ }^{15}$ and disruption of the RHT results in an inability to respond to light as a circadian resetting/entraining signal. ${ }^{16,17}$ Recent work has found that many of the retinal ganglion cells that comprise the RHT contain a photopigment, melanopsin. ${ }^{18}$

These melanopsin-containing cells show a response to light that parallels circadian responses to light. ${ }^{19}$ Additionally, the terminals of the melanopsinpositive retinal ganglion cells collocalize both glutamate and pituitary adenylate cyclase-activating polypeptide (PACAP), ${ }^{20}$ the putative neurotransmitters of the RHT.

The geniculohypothalamic tract (GHT) also appears to play a role in transmitting light information to the clock. The GHT projects from the intergeniculate leaflet (IGL) to the SCN, and releases neuropeptide Y (NPY). NPY is believed to be involved in behavior-induced phase shifts during the daytime, but also appears to be able to modulate light-induced phase shifts. ${ }^{21,22}$ However, while the GHT pathway can transmit photic signals, disruption of this pathway does not prevent entrainment to light. ${ }^{23}$

The SCN also receives serotonergic input, primarily from the median raphe. Activation of the median raphe results in an increase in serotonin (5-HT) release at the $\mathrm{SCN} .{ }^{24} 5$-HT release also shows a strong circadian pattern in the SCN, with 5-HT release peaking at CT 14, and 5-hydroxyindole acetic acid (5-HIAA), the major metabolite of 5-HT peaking at CT $16 .{ }^{25}$

Cholinergic projections to the SCN originate both in the basal forebrain and in the laterodorsal tegmental (LDT), pedunculopontine tegmental (PPT) and parabigeminal nuclei $(\mathrm{PBg})$ in the brainstem. ${ }^{26}$ The $\mathrm{PBg}$ is considered a satellite region of the superior colliculus, which appears to play a role in generating target-location information as part of saccadic eye-movements. ${ }^{27}$ 
The substantia innominata (SI) within the nucleus basalis magnocellularis (NBM) in the basal forebrain contributes to arousal and focused attention, ${ }^{28}$ while the LDT and PPT both are important for regulating the sleep-wake cycle. ${ }^{29}$ This would suggest that the cholinergic input to the $\mathrm{SCN}$ is providing a signal regarding the sleep-wake state of the animal, and may provide a link between the sleep-wake cycle and circadian rhythms.

\section{Circadian Clock Regulators}

With the anatomy in place, we now can begin looking at how the SCN responds to various inputs. One model that works particularly well for studying the signaling mechanisms that regulate circadian rhythms is to examine the electrophysiological properties of a hypothalamic brain slice preparation containing the SCN. Coronal slices of hypothalamus containing the SCN can be maintained at the interface of a warm, moist, high oxygen atmosphere and a solution containing minimal salts and glucose for at least 3 days, and will show a peak in neuronal firing activity that occurs at approximately the same time every day (Figure 2). ${ }^{30,31}$

Using this preparation, the pattern and rate of extracellular activity of individual neurons within the SCN can be recorded. When data from individual neurons are averaged over circadian time of recording, a peak in average firing rate of the neuronal ensemble occurs at approximately CT 7 .

Using this as a baseline marker of circadian time in the brain slice, experiments can then be performed where neurotransmitters or candidate elements in a signaling pathway can be applied to the slice at different times of the day and the resultant change in time of peak can be measured to determine the effect on SCN circadian rhythms. This generates the PRC of the isolated $\mathrm{SCN}$ for each substance.

This system is very versatile, and it can be used to examine the effect of different agonists on resetting circadian rhythms, as well as whether antagonists of putative downstream members of the signaling pathway can block the normally observed effects. While the SCN appears to be involved in sending signals as to time of day to the rest of the body, the fact that the isolated preparation expresses differing sensitivities to resetting signals that are dependent on circadian time demonstrates that the SCN itself is intrinsically sensitive to time of day. We will now examine the various resetting pathways that are present in the SCN, focusing on the different pathways that can regulate the clock during the different times of the day.

\subsection{Daytime}

A number of signaling molecules appear to be important in resetting circadian rhythms during the daytime, including 5-HT, PACAP, NPY and GABA. The majority of these experiments have been performed in nocturnal rodents, 


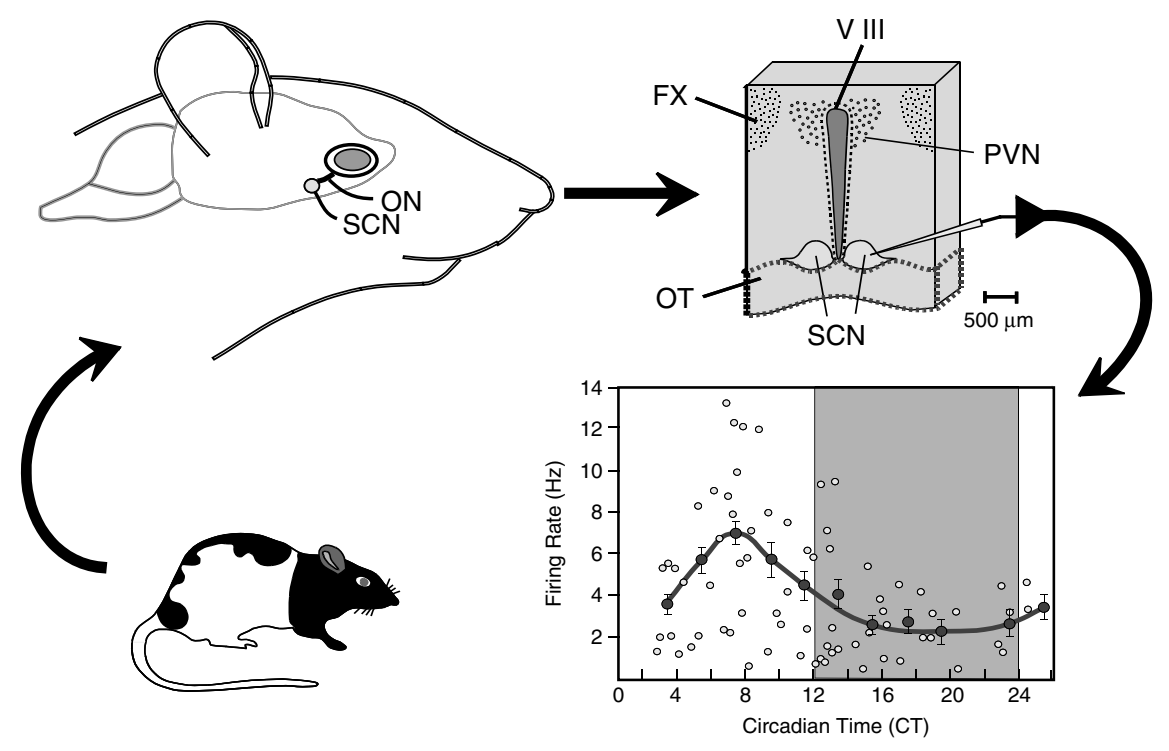

FIGURE 2. A diagrammatical representation of the hypothalamic brain slice preparation. A coronal slice of the hypothalamus is removed from the Long Evans BluGill rat, placed in culture, and extracellular neuronal activity is recorded. A representative plot of individual neuron activity along with successive 2-h means are plotted, as indicated by the line. This preparation can be maintained for at least 3 days in vitro, producing the characteristic peak in firing rate at $\sim \mathrm{CT} 7$ each day. ON/OT, optic nerve/tract; VIII, third cerebral ventricle; open circles, single unit activity; closed circles, 2-h mean ensemble activity; shading CT 12-24, subjective night.

so daytime is defined as the time in which the lights are on and/or the animal is inactive. As a result, the functional context of this regulation seems to be tied to non-photic (non-light stimulated) resetting. Non-photic resetting covers a wide variety of resetting phenomenon, including sleep deprivation, activity associated with exposure to a novel wheel and even cage changes. The unifying factor in non-photic resetting is that it involves arousal during a time when the animal would normally be inactive. Many of the neurotransmitters that have been linked experimentally with this daytime resetting also appear to modulate nighttime resetting. Daytime effects will be discussed here, while the modulation of nighttime effects will be covered in the next section.

While 5-HT is believed to play a role in activity-induced or non-photic phase shifts during the day, there is some question about whether this form of phase shifting is entirely due to 5-HT. If serotonergic agonists are applied to the hypothalamic brain slice during the daytime, the peak in electrical firing activity advances, but no change in time of peak firing rate is seen if the agonists are applied during the night (Figure 3). ${ }^{32}$ Similar results are seen 


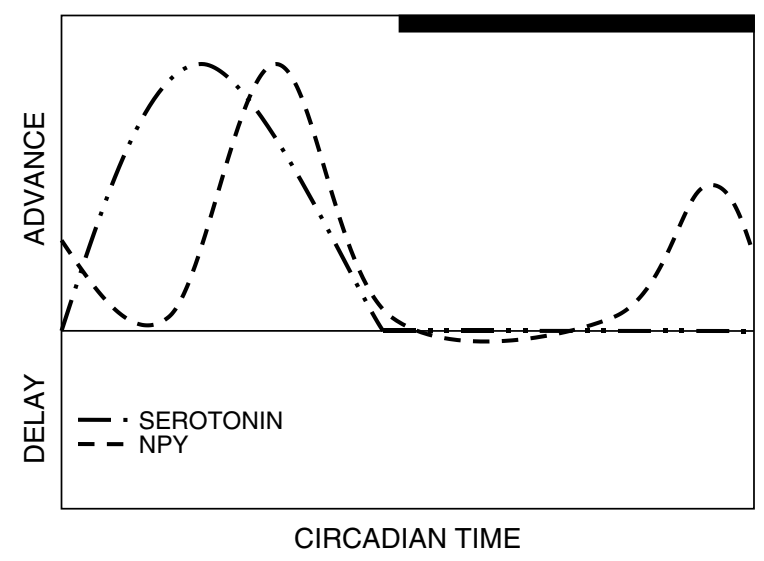

FigURE 3. Summary of daytime circadian resetting signals. This figure depicts the in vitro response of the $\mathrm{SCN}$ to serotonin and neuropeptide $\mathrm{Y}$, which act on the circadian clock during daytime. The circadian time of treatment is represented on the $\mathrm{X}$-axis, while the magnitude of advance or delay is represented on the Y-axis. Time in which the animal would be in darkness is represented by a black bar. As can be seen, each neurotransmitter has a unique resetting profile, both in time and direction of response throughout the day. Replotted from M. Medanic and M. U. Gillette, Serotonin regulates the phase of the rat suprachiasmatic circadian pacemaker in vitro only during the subjective day. J. Physiol. 450, 629-642 (1982) and M. Medanic and M. U. Gillette, Suprachiasmatic circadian pacemaker of rat shows two windows of sensitivity to neuropeptide Y in vitro. Brain Res. 620:281-286 (1993).

in vivo if the dorsal or medial raphe is stimulated, ${ }^{33}$ a paradigm that has been shown by microdialysis to increase 5 -HT release at the level of the SCN. ${ }^{34}$ During forced wheel running or sleep deprivation during the daytime, there is an increase in 5-HT release at the SCN. ${ }^{35,36}$ This suggests a link between 5HT and non-photic phase shifting, but evidence also exists to complicate this assertion. If $85-95 \%$ of the serotonin is depleted from the raphe projections to the SCN, animals still are capable of phase shifting in response to daytime forced activity. ${ }^{37}$ In addition, these activity-induced phase shifts are not significantly attenuated following injection of serotonergic antagonists. ${ }^{38}$ These data suggest that, while 5-HT may play a role in non-photic resetting, the full resetting response depends on modulation by additional neurotransmitters.

PACAP appears to play a dual role in the SCN, producing effects both during the daytime and at night, when it acts in conjunction with glutamate. PACAP is released from the RHT, and studies have found that it is colocalized with glutamate. ${ }^{39}$ Examining levels of PACAP throughout the 24-h cycle revealed that PACAP exhibits a significant oscillation in the $\mathrm{SCN}$, but not in other brain regions, and is lower during the light period than the dark period. ${ }^{40}$ If PACAP is applied to the brain slice at different times of day, 
micromolar quantities will cause an advance in neuronal firing activity during the daytime, but have relatively little effect during the night. ${ }^{20}$ However, when PACAP is injected into the SCN of the hamster between CT 4-8, transient phase advances in wheel-running activity are seen during the first day after treatment, but the long-term effects of a PACAP injection appear to produce a delay in wheel-running activity. ${ }^{41}$ This suggests that, while PACAP has an effect on circadian rhythms during the daytime, further work is needed to determine the precise nature of this signal.

NPY also appears to play a dual role, and is effective in resetting the circadian clock both during the daytime and at night. NPY is released from the GHT, the projection from the IGL of the thalamus to the SCN. Studies have examined the effects of either injecting NPY into the SCN region of the intact animal and monitoring wheel running behavior ${ }^{42,43}$ or applying NPY directly to the hypothalamic brain slice and examining the peak in neuronal firing activity. ${ }^{21}$ In both cases it was found that when NPY was applied during the daytime, it induced an advance in the circadian parameter of interest (Figure 3). Additional studies implanted stimulating electrodes into the IGL, which would presumably release NPY at the SCN when activated, and examined the effects of IGL stimulation on circadian wheel-running behavior. ${ }^{44}$ These stimulations also produced advances in circadian phase when given during the daytime. Interestingly, it has been found that exposing an animal to light ${ }^{45}$ or applying glutamate to the brain slice ${ }^{46}$ both were capable of blocking the response to daytime application of NPY. Co-administration of the GABA antagonist, bicuculline, is capable of inhibiting the effects of NPY in vivo, ${ }_{A} 7$ suggesting that the NPY signal is linked to GABAergic signaling.

One factor that daytime signaling pathways hold in common is that they all appear to be mediated by cyclic adenosine monophosphate (cAMP). In the hypothalamic brain slice, cAMP analogs applied during the daytime induce phase advances in the circadian clock, while at night they have little effect. ${ }^{31,48}$ In addition, endogenous cAMP is high during late day, and late night, ${ }^{49}$ suggesting a role for cAMP in the transition periods between day and night, and that induction of a rise in cAMP may move the clock to a time-point where cAMP is normally high.

\subsection{Dawn and Dusk}

The primary resetting signal associated with dawn and dusk is melatonin. This hormone of darkness is produced at night in the absence of light, providing a means by which the animal can measure night-length. ${ }^{50}$ Photoperiod is an important measure for animals, such as the hamster, that are seasonally reproductive.

Melatonin is produced by the pineal, and in many vertebrates the pineal is actually the primary regulator of circadian rhythms, rather than the SCN. However, in mammals, removal of the pineal does not significantly disrupt circadian rhythms. ${ }^{51}$ 
Despite the fact that a pineal is not necessary for maintenance of mammalian circadian rhythms, it is possible to entrain free-running rats with daily injections of melatonin. Entrainment appears to work best if the melatonin injections are timed to occur shortly before the onset of the animal's active period. In addition, lesioning the SCN, but not the pineal, abolishes the ability of a rat to entrain to melatonin injections. ${ }^{52}$

Evidence that melatonin can reset circadian rhythms led to a number of studies looking at the direct effect of melatonin on the SCN. Using either 2-deoxy-glucose (2-DG) or neuronal activity as a marker of SCN activity, it was found that melatonin decreased both 2-DG uptake and neuronal firing activity in the rat most significantly when applied right before subjective dusk..$^{53,54}$ Melatonin-induced suppression of neuronal firing activity at the end of the day also is seen in the hamster. ${ }^{55}$

The SCN shows a similar window of melatonin sensitivity in terms of resetting the circadian clock. Using the hypothalamic brain slice, it was found that melatonin applied at either dawn or dusk advanced the peak in neuronal firing, but when applied at other times of day no effect was observed (Figure 4). ${ }^{56,57}$ This resetting pattern mimicked that seen in response to activation of protein kinase $\mathrm{C}$ (PKC), and was blocked by inhibitors of PKC, suggesting that $\mathrm{PKC}$ was a downstream component of this resetting pathway.

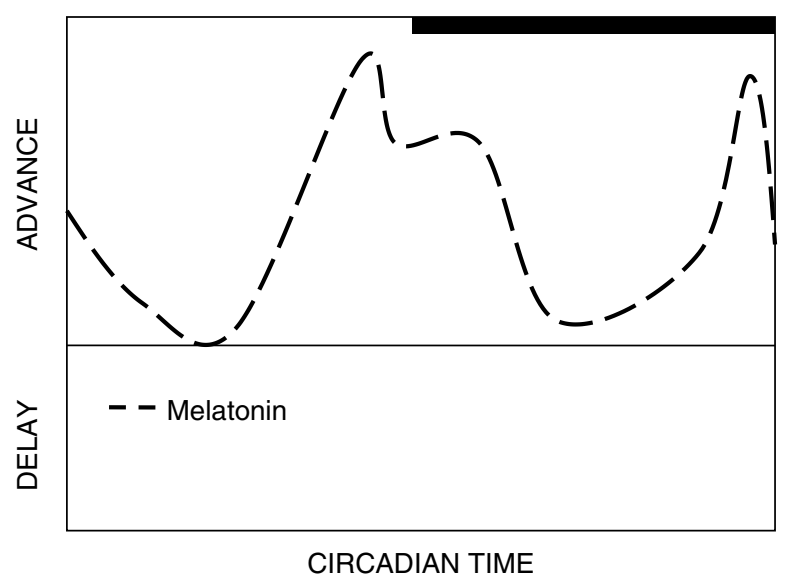

FIGURE 4. Summary of dawn/dusk circadian resetting signals. This figure depicts the in vitro response of the $\mathrm{SCN}$ to melatonin. The circadian time of treatment is represented on the $\mathrm{X}$-axis, while the magnitude of advance or delay is represented on the Y-axis. Time in which the animal would be in darkness is represented by a black bar. As can be seen, this neurohormone has a unique resetting profile, both in time and direction of response at the subjective day- to night and night-to-day transitions. Replotted from A. J. McArthur, A. E. Hunt, and M. U. Gillette, Melatonin action and signal transduction in the rat suprachiasmatic circadian clock: activation of protein kinase C at dusk and dawn. Endocrinology 138(2), 627-634 (1997). 
In addition, this resetting could be inhibited with antagonists that were specific for the MT-2 type melatonin receptor. ${ }^{58}$

\subsection{Nighttime}

In the nighttime domain there are two key players, glutamate and $\mathrm{ACh}$, as well as a number of substances that modulate these signals. As we have discussed previously, considerable evidence supports glutamate as the primary neurochemical signal transmitting photic stimuli from the retina to the $\mathrm{SCN}$, while the functional context of the cholinergic resetting signal is still unknown.

The glutamate signaling pathway is similar to many of the pathways that have already been discussed, in that it will reset the circadian clock at a particular time of day, and in a specific direction. However, what is interesting about the glutamate signaling pathway is that it can either advance or delay the clock, depending on what time of day the signal is presented (Figure 5). ${ }^{59}$

This pathway has been demonstrated both in vitro and in vivo to be mediated through an N-methyl-D-aspartate (NMDA) receptor-mediated rise in

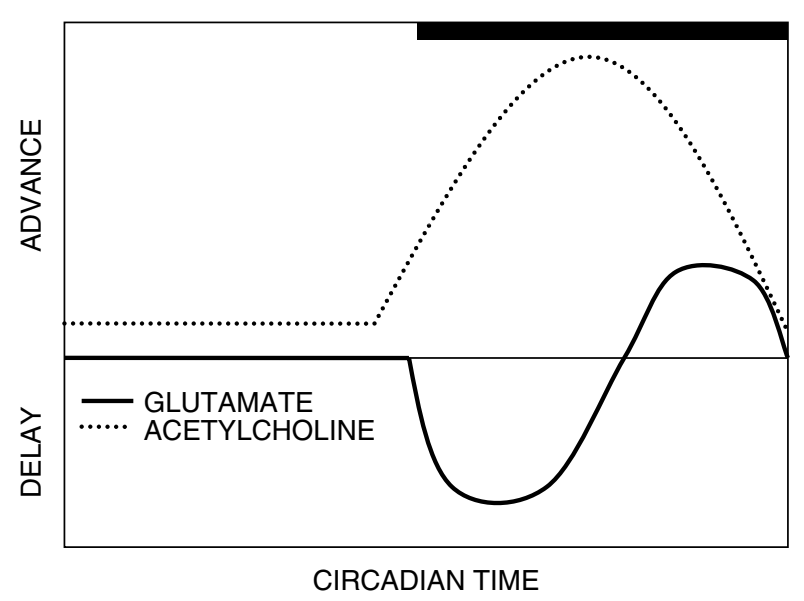

FIGURE 5. Summary of nighttime circadian resetting signals. This figure depicts the in vitro response of the SCN to glutamate and accetylcholine, neurotransmitters effective primarily at night. The circadian time of treatment is represented on the $\mathrm{X}$-axis, while the magnitude of advance or delay is represented on the Y-axis. Time in which the animal would be in darkness is represented by a black bar. As can be seen, each neurotransmitter has a unique resetting profile, both in time and direction of response throughout the night. Replotted from C. Liu and M. U. Gillette, Cholinergic regulation of the suprachiasmatic nucleus circadian rhythm via a muscarinic mechanism at night. J. Neurosci. 16(2), 744-751 (1996) and J. M. Ding, D. Chen, E. T. Weber, et al., Resetting the biological clock: mediation of nocturnal circadian shifts by glutamate and NO. Science 266, 1713-1717 (1994). 
intracellular calcium, followed by nitric oxide synthase (NOS) activation and resultant production of nitric oxide (NO). ${ }^{60-64}$ Beyond this point, the early- and late-night pathways diverge.

During the early night, glutamate induces delays in the circadian clock. Downstream of NO, the next step in this pathway appears to be ryanodine receptor (RyR)-mediated calcium release. In the brain slice, pharmacological activation of the RyR induces a phase delay in neuronal activity during the early night, but not at any other time of day. This effect can be blocked both in vitro and in vivo with dantrolene, an RyR inhibitor. ${ }^{65}$

Glutamate exposure during the late night, however, advances the circadian clock. During the late night, NO appears to activate a cyclic guanosine monophosphate/protein kinase G (cGMP/PKG) signaling cascade followed by cAMP response element binding protein (CREB)-activated transcription. Inhibiting $\mathrm{PKG}$ in the brain slice blocks light-induced advances in neuronal activity. ${ }^{65}$ Phospho-CREB (P-CREB) is induced in the SCN following exposure to light at night, while antagonists of putative elements of the upstream signaling pathway, such as NMDA or NOS, inhibit the induction of P-CREB. ${ }^{66}$ Furthermore, adding an excess oligodeoxynucleotide decoy sequence that inhibits cAMP response element (CRE)-mediated transcription blocks the phase advance induced by glutamate both in vitro and in vivo. ${ }^{67}$

While glutamate alone is capable of resetting circadian rhythms, there are many substances that modulate this resetting. These can be divided into two categories: those that decrease the phase resetting effect of glutamate during both the early and late night, and those that have differing effects on glutamate-induced phase shifts, depending on what time of night they are applied. In the first category, both NPY and GABA, which are active in daytime phase shifting, decrease the phase-resetting effect of light or glutamate when applied during the night. ${ }^{68,69}$ Glutamate applied during the daytime also seems to be capable of inhibiting GABA- and serotonin-induced resetting. ${ }^{69,70}$

Modulatory effects of serotonin and PACAP on glutamate-induced phase resetting are more complicated. If animals are depleted of serotonin, they show increased phase delays in response to light. ${ }^{71}$ On the other hand, coapplication of a PACAP antagonist, either in vitro or in vivo, decreases the phase delay seen in early night, and increases the late-night phase advance. $^{72,73}$

When PACAP is administered in conjunction with glutamate, it increases the early-night phase delays, but decreases the late-night phase advances. This is similar to the effects seen following application of cAMP analogs to the hypothalamic brain slice, suggesting that the effects of PACAP may be mediated by a cAMP pathway. ${ }^{74}$

The role of $\mathrm{ACh}$ in resetting circadian rhythms is rather complicated, and has been unclear, because the effect varies with the site of application. The first evidence that ACh might play a role in resetting the circadian clock came 
in 1979, when Zatz and Brownstein examined whether pharmacological manipulation of the $\mathrm{SCN}$ could affect serotonin $\mathrm{N}$-acetyltransferase (SNAT) activity in the pineal. ${ }^{75}$

They found that injections of carbachol into the lateral ventricle of Sprague-Dawley rats at CT 15 caused phase delays in SNAT activity that were similar to, but not as large as, the phase delays produced by light. ${ }^{75}$ Carbachol injections into the lateral ventricle were also later repeated in mice ${ }^{76}$ and hamsters, ${ }^{77}$ where it was found that administration of carbachol during the early night caused phase delays, while late-night administration caused phase advances. This pattern of sensitivity and response is similar to that induced by light exposure. Further evidence for the involvement of $\mathrm{ACh}$ in the light response came from studies looking at ACh levels in the rat $\mathrm{SCN}$ using a radioimmunoassay (RIA). ${ }^{78}$ Using this technique, no significant oscillation in ACh levels was found under constant conditions, but light pulses administered at CT 14 increased ACh levels in the SCN. However, no work was done to determine whether this increase was simply a response to exposure to light or if there was actually a circadian pattern to the light-stimulated ACh release. The implication of these studies was that ACh could be the primary neurotransmitter providing the signal of light to the clock.

However, significant evidence began to emerge indicating that ACh was not likely to be the primary signal of light. First of all, whereas it had previously been determined that the RHT transmitted the signal of light from the eye to the SCN, choline acetyltransferase (ChAT) was not present in this projection, ${ }^{79}$ making it anatomically unlikely that ACh was the primary neurotransmitter involved in this signal. This evidence can be reconsidered, however, as recent studies have found an alternative splice variant of ChAT present in retinal ganglion cells that was not picked up using previous antibodies ${ }^{80}$ Experiments have not yet been published addressing whether this alternative form of ChAT is present in the RHT.

Additional evidence against ACh being the signal of light came from experiments that found injecting hemicholinium, which significantly depletes ACh stores, did not block the ability of the animal to phase shift in response to light. ${ }^{81}$ There is also evidence that injecting NMDA receptor antagonists could block carbachol-induced phase shifts, suggesting that although ACh may play a role in the light response, it is not the direct cause of the observed phase shifts. ${ }^{82}$ Finally, Liu and Gillette using extracellular recording in vitro, found that microdrop applications of carbachol directly to the SCN caused only phase advances, regardless of whether it was applied early or late in subjective nighttime (Figure 5). ${ }^{83}$

In an attempt to explain these contradicting data, the hypothesis that our lab has developed is that the dual response pattern of the SCN to cholinergic stimulation is a result of the location of application. Note that in the initial in vivo studies, carbachol was injected into the lateral or third ventricle, where the drug could have a diffuse effect, while in the in vitro studies, carbachol was 
applied in microdrops directly to the SCN. Emerging evidence suggests that, if the in vivo experiments are performed by injecting carbachol directly into the SCN rather than into the ventricle, a similar phase-response pattern is seen as resulted from the in vitro experiments using microdrop applications. ${ }^{84}$ Furthermore, preliminary studies suggest that mice lacking the $\mathbf{M}_{1}$-type muscarinic receptor $\left(\mathrm{M}_{1} \mathrm{AChR}\right)$ do not respond to these carbachol injections, ${ }^{85}$ but exhibit normal responses to light. ${ }^{86}$ Together, the evidence indicates that, while ACh may contribute to the light response, as seen in earlier experiments, the direct cholinergic signal to the $\mathrm{SCN}$ is providing an entraining signal that is different than the signal of light, and this response is mediated by an $\mathrm{M}_{1} \mathrm{AChR}$. Based on the anatomical studies looking at cholinergic projections to the SCN that originate in the LDT and PPT, as well as the SI, the current hypothesis is that this cholinergic signal is involved in tying the sleep-wake and circadian cycles together.

\section{Circadian and Sleep/Wake Cycle Interactions}

\subsection{The Two-Process Mode of Sleep and Circadian Interactions}

The circadian and sleep-wake cycles have been known to interact. This interaction was modeled by Borbely as regulated by two processes: process $\mathrm{S}$ and process C. Process S is sleep-dependent; its influence becomes greater the longer one has been without sleep. Process $\mathrm{C}$ is circadian-dependent; it varies with circadian phase of the day or night.$^{87}$ Process $\mathrm{S}$ uses as its marker electroencephalographic (EEG) slow-wave activity, which may be promoted by accumulation of adenosine. During the time awake, the rising homeostatic sleep pressure from process $\mathrm{S}$ is compensated for by a decline in circadian process $\mathrm{C}$ sleep pressure. ${ }^{88}$ The converse is true at night. The interaction between the two processes accounts for both the consolidation of sleep and wake under normal conditions, as well as the fact that there is a rhythmic variation in sleep propensity throughout the duration of sleep deprivation. The interaction between these two processes can be further demonstrated by looking at what happens to one process in the absence of the other, as will be described in the next section.

\subsection{Sleep and Circadian Interactions}

Initial studies evaluating the interaction between the circadian and sleep-wake cycles simply determined the effect of SCN lesions on circadian rhythms. Bilateral SCN lesions in rat were found to eliminate the circadian rhythm of the sleep-wake cycle. ${ }^{89}$ While total time spent in sleep remained the same, the occurrence of sleep was now randomly distributed between the light and dark phases, rather than ocurring primarily during daytime. ${ }^{89}$ However, the 
ultradian rhythm of slow wave sleep (SWS) cycles was still present..$^{90}$ Later studies found a slight increase in total SWS and slight decrease in total rapid eye movement (REM) sleep in SCN-lesioned rats. ${ }^{91}$

The next step was to look at whether circadian inputs influenced the homeostatic response, by looking at how SCN-lesioned animals responded to periods of sleep deprivation. Initially, these animals were found to still show a homeostatic recovery response, but on further study, differences were found in the speed of recovery under different conditions. ${ }^{92}$ Animals that contained an intact SCN and were placed under constant lighting conditions showed the fastest recovery from a period of sleep-deprivation. Animals with SCNlesions or on a regular light-dark cycle required more time than controls to recover the lost sleep, suggesting that an intact circadian system, without the influence of outside time cues such as light, provides the most efficient means of recovering lost sleep. ${ }^{93}$ Although squirrel monkeys exhibited slightly different patterns of response than rodents to SCN lesions followed by sleep deprivation, in that their total sleep time increases, the total time spent in NREM and REM sleep did not increase. ${ }^{94}$

Feedback from the sleep-wake cycle to the circadian clock was evaluated by looking at the effect of sleep deprivation and arousal on circadian rhythms. If hamsters are sleep-deprived during the latter part of their active period, either through exposure to a novel wheel, or by gentle handling, they exhibit a dramatic advance in onset of wheel-running activity that remains stable as long as they remain in constant conditions. ${ }^{36,95}$ This sleepdeprivation paradigm has also been used in mice to demonstrate that the phase delays observed in response to an early night light pulse are actually decreased if the animal has been sleep-deprived prior to the light pulse. ${ }^{96}$

A recent study provided a more functional examination of this feedback loop by examining the correlation between sleep-wake state and neuronal firing rate in the SCN. Early work found that SCN cells undergo changes in firing rate that relate to arousal state of the animal. ${ }^{97}$ More recent studies conducted a detailed analysis of SCN neuronal activity during different sleep stages, and found that the SCN firing rate exhibited the previously observed diurnal pattern, but superimposed on it was a change in firing rate dependent on sleep state of the animal. During wake or REM sleep, SCN firing rates were much higher than during NREM sleep. ${ }^{98}$ As discussed earlier in this chapter, there are circuits in place for most sleep centers in the brain to feed back to the SCN, and these studies provide evidence that this feedback actually has functional consequences.

\section{Conclusion}

Circadian rhythms are an integral part of an organism's existence. In mammals, these rhythms are regulated by a circadian clock in the SCN, which is uniquely situated to receive a variety of inputs regarding the animal's 
environment and behavioral state. Of particular relevance for this chapter is the fact that the circadian system plays an important role in regulating when an animal is active and inactive. There is evidence that the SCN sends efferents to parts of the brain involved in arousal, and that in the absence of a circadian clock, consolidation of the sleep-wake cycle disappears. In addition, recent studies suggest that the sleep-wake state of the animal is capable of feeding back to the circadian clock. Sleep deprivation of hamsters at the appropriate time of day can reset the phase of the circadian clock, and changes in sleep-wake state appear to influence the firing rate of neurons within the SCN. Overall, evidence indicates that the circadian and sleep-wake cycles are intricately intertwined, and that study of one necessitates the study and consideration of the other.

5. Acknowledgments. Thanks to past and present members of the Gillette lab for contributions to studies on which this chapter is based, and to S. C. Baker for manuscript preparation. Supported by Public Health Service Grants NS22155, NS35859 and HL67007 (MUG) and NIH Training Grant GM07143 (SMA). Any opinions, findings, and conclusions or recommendations expressed in this publication are those of the author and do not necessarily reflect the views of the National Institutes of Neurological Diseases \& Stroke, Heart, Lung, and Blood, or General Medicine.

\section{References}

1. R. M. Buijs, C. G. van Eden, V. D. Goncharuk and A. Kalsbeek, The biological clock tunes the organs of the body: timing by hormones and the autonomic nervous system. J. Endocrinol. 177, 17-26 (2003).

2. C. Cajochen, K. Krauchi and A. Wirz-Justice, Role of melatonin in the regulation of human circadian rhythms and sleep. J. Neuroendocrinol. 15, $432-437$ (2003).

3. S. E. la Fleur, Daily rhythms in glucose metabolism: suprachiasmatic nucleus output to peripheral tissue. J. Neuroendocrinol. 15, 315-322 (2003).

4. F. K. Stephan and I. Zucker, Circadian rhythms in drinking behavior and locomotor activity of rats are eliminated by hypothalamic leions. Proc. Natl. Acad. Sci. U.S. A. 69, 1583-1586 (1972).

5. R. Y. Moore and V. B. Eichler, Loss of a circadian adrenal corticosterone rhythm following suprachiasmatic lesions in the rat. Brain Res. 42, 201-206 (1972).

6. R. Drucker-Colin, R. Aguilar-Roblero, F. Garcia-Hernandez, F. FernandezCancino and F. B. Rattoni, Fetal suprachiasmatic nucleus transplants: diurnal rhythm recovery of lesioned rats. Brain Res. 311, 353-357 (1984).

7. M. R. Ralph, R. G. Foster, F. C. Davis and M. Menaker, Transplanted suprachiasmatic nucleus determines circadian period. Science 247, 975-978 (1990).

8. R. Y. Moore, J. C. Speh and R. K. Leak, Suprachiasmatic nucleus organization. Cell Tissue Res. 309, 89-98 (2002).

9. R. K. Leak and R. Y. Moore, Topographic organization of suprachiasmatic nucleus projection neurons. J. Compara. Neurol. 433, 312-334 (2001). 
10. E. E. Abrahamson, R. K. Leak and R. Y. Moore, The suprachiasmatic nucleus projects to posterior hypothalamic arousal systems. Neuroreport 12(2), 435-440 (2001).

11. T. C. Chou, A. A. Bjorkum, S. E. Gaus, J. Lu, T. E. Scammell and C. B. Saper, Afferents to the ventrolateral preoptic nucleus. J. Neurosci. 22(3), 977-990 (2002).

12. A. Kalsbeek and R. M. Buijs, Output pathways of the mammalian suprachiasmatic nucleus: coding circadian time by transmitter selection and specific targeting. Cell Tissue Res. 309, 109-118 (2002).

13. A. Kramer, F.-C. Yang, P. Snodgrass, et al., Regulation of daily locomotor activity and sleep by hypothalamic EGF receptor signaling. Science 294, 2511-2515 (2001).

14. M. Y. Cheng, C. M. Bullock, C. Li, et al., Prokineticin 2 transmits the behavioural circadian rhythm of the suprachiasmatic nucleus. Nature 417, 405-410 (2002).

15. S. Mosko and R. Moore, Retinohypothalamic tract development: alteration by suprachiasmatic lesions in the neonatal rat. Brain Res. 164, 1-15 (1979).

16. B. Rusak, Neural mechanisms for entrainment and generation of mammalian circadian rhythms. Fed. Proc. 38(12), 2589-2595 (1979).

17. R. F. Johnson, R. Y. Moore and L. P. Morin, Loss of entrainment and anatomical plasticity after lesions of the hamster retinohypothalamic tract. Brain Res. 460, 297-313 (1988).

18. S. Hattar, R. J. Lucas, N. Mrosovsky, et al., Melanopsin and rod-cone photoreceptive systems account for all major accessory visual functions in mice. Nature 424, 76-81 (2003).

19. D. M. Berson, F. A. Dunn and M. Takao, Phototransduction by retinal ganglion cells that set the circadian clock. Science 295, 1070-1073 (2002).

20. J. Hannibal, J. M. Ding, D. Chen, et al., Pituitary adenylate cyclase-activating peptide (PACAP) in the retinohypothalamic tract: a potential daytime regulator of the biological clock. J. Neurosci. 17, 2637-2644 (1997).

21. M. Medanic and M. U. Gillette, Suprachiasmatic circadian pacemaker of rat shows two windows of sensitivity to neuropeptide $\mathrm{Y}$ in vitro. Brain Res. 620, 281286 (1993).

22. P. C. Yannielli and M. E. Harrington, The neuropeptide Y Y5 receptor mediates the blockade of "photic-like" NMDA-induced phase shifts in the golden hamster. J. Neurosci. 21(14), 5367-5373 (2001).

23. V. Reghunandanan, R. Reghunandanan and P. I. Singh, Neurotransmitters of the suprachiasmatic nucleus: role in the regulation of circadian rhythms. Prog. Neurobiol. 41, 647-655 (1993).

24. A. N. Van den Pol and K. L. Tsujimoto, Neurotransmitters of the hypothalamic suprachiasmatic nucleus: immunocytochemical analysis of 25 neuronal antigens. Neuroscience 15, 1049-1086 (1985).

25. S. Barassin, S. Raison, M. Saboureau, et al., Circadian tryptophan hydroxylase levels and serotonin release in the suprachiasmatic nucleus of the rat. Eur. J. Neurosci. 15, 833-840 (2002).

26. K. G. Bina, B. Rusak and K. Semba, Localization of cholinergic neurons in the forebrain and brainstem that project to the suprachiasmatic nucleus of the hypothalamus in rat. J. Compara. Neurol. 335, 295-307 (1993).

27. H. Cui and J. G. Malpeli, Activity in the parabigeminal nucleus during eye movements directed at moving and stationary targets. J. Neurophysiol. 89(6), 3128-3142 (2003). 
28. K. Semba, Multiple output pathways of the basal forebrain: organization, chemical heterogeneity, and roles in vigilance. Behav. Brain Res. 115, 117-141 (2000).

29. S. Deurveilher and E. Hennevin, Lesions of the pedunculopontine tegmental nucleus reduce paradoxical sleep (PS) propensity: evidence from a short-term PS deprivation study in rats. Eur. J. Neurosci. 13, 1963-1976 (2001).

30. D. J. Green and R. Gillette, Circadian rhythm of firing rate recorded from single cells in the rat suprachiasmatic brain slice. Brain Res. 245, 198-200 (1982).

31. R. A. Prosser and M. U. Gillette, The mammalian circadian clock in the suprachiasmatic nuclei is reset in vitro by cAMP. J. Neurosci. 9(3), 1073-1081 (1989).

32. M. Medanic and M. U. Gillette, Serotonin regulates the phase of the rat suprachiasmatic circadian pacemaker in vitro only during the subjective day. J. Physiol. 450, 629-642 (1992).

33. J. D. Glass, L. A. DiNardo and J. C. Ehlen, Dorsal raphe nuclear stimulation of SCN serotonin release and circadian phase-resetting. Brain Res. 859, 224-232 (2000).

34. T. E. Dudley, L. A. Dinardo and J. D. Glass, In vivo assessment of the midbrain raphe nuclear regulation of serotonin release in the hamster suprachiasmatic nucleus. J. Neurophysiol. 81(4), 1469-1477 (1999).

35. T. E. Dudley, L. A. DiNardo and J. D. Glass, Endogenous regulation of serotonin release in the hamster suprachiasmatic nucleus. J. Neurosci. 18(13), 5045-5052 (1998).

36. G. H. Grossman, R. E. Mistlberger, M. C. Antle, J. C. Ehlen and J. D. Glass, Sleep deprivation stimulates serotonin release in the suprachiasmatic nucleus. Neuroreport 11(9), 1929-1932 (2000).

37. K. J. Bobrzynska, N. Vrang and N. Mrosovsky, Persistence of nonphotic phase shifts in hamsters after serotonin depletion in the suprachiasmatic nucleus. Brain Res. 741(1-2), 205-214 (1996).

38. M. C. Antle, E. G. Marchant, L. Niel and R. E. Mistlberger, Serotonin antagonists do not attenuate activity-induced phase shifts of circadian rhythms in the Syrian hamster. Brain Res. 813, 139-149 (1998).

39. J. Hannibal, M. Moller, O. P. Ottersen and J. Fahrenkrug, PACAP and glutamate are co-stored in the retinohypothalamic tract. J. Compara. Neurol. 418, 147-155 (2000).

40. C. Fukuhara, N. Suzuki, Y. Matsumoto, et al., Day-night variation of pituitary adenylate cyclase-activating polypeptide (PACAP) level in the rat suprachiasmatic nucleus. Neurosci. Lett. 229, 49-52 (1997).

41. H. D. Piggins, E. G. Marchant, D. Goguen and B. Rusak, Phase-shifting effects of pituitary adenylate cyclase activating polypeptide on hamster wheel-running rhythms. Neurosci. Lett. 305, 25-28 (2001).

42. H. E. Albers and C. F. Ferris, Neuropeptide Y: role in light-dark cycle entrainment of hamster circadian rhythms. Neurosci. Lett. 50, 163-168 (1984).

43. K. L. Huhman and H. E. Albers, Neuropeptide Y microinjected into the suprachiasmatic region phase shifts circadian rhythms in constant darkness. Peptides 15(8), 1475-1478 (1994).

44. B. Rusak, J. H. Meijer and M. E. Harrington, Hamster circadian rhythms are phase-shifted by electrical stimulation of the geniculo-hypothalamic tract. Brain Res. 493, 283-291 (1989). 
45. S. M. Biello and N. Mrosovsky, Blocking the phase-shifting effect of neuropeptide Y with light. Proc. R. Soc. Lond., B, Biol. Sci. 259, 179-187 (1995).

46. S. M. Biello, D. A. Golombek and M. E. Harrington, Neuropeptide Y and glutamate block each other's phase shifts in the suprachiasmatic nucleus in vitro. Neuroscience 77(4), 1049-1057 (1997).

47. K. L. Huhman, T. O. Babagbemi and H. E. Albers, Bicuculline blocks neuropeptide Y-induced phase advances when microinjected in the suprachiasmatic nucleus of syrian hamsters. Brain Res. 675, 333-336 (1995).

48. M. U. Gillette and R. A. Prosser, Circadian rhythm of the rat suprachiasmatic brain slice is rapidly reset by daytime application of cAMP analogs. Brain Res. 474, 348-352 (1988).

49. R. A. Prosser and M. U. Gillette, Cyclic changes in cAMP concentration and phosphodiesterase activity in a mammalian circadian clock studied in vitro. Brain Res. 568, 185-192 (1991).

50. M. U. Gillette and A. J. McArthur, Circadian actions of melatonin at the suprachiasmatic nucleus. Behav. Brain Res. 73, 135-139 (1996).

51. P. W. Cheung and C. E. McCormick, Failure of pinealectomy or melatonin to alter circadian activity rhythm of the rat. Am. J. Physiol. 242, R261-R264 (1982).

52. V. M. Cassone, M. J. Chesworth and S. M. Armstrong, Entrainment of rat circadian rhythms by daily injection of melatonin depends upon the hypothalamic suprachiasmatic nuclei. Physiol. Behav. 36, 1111-1121 (1986).

53. V. M. Cassone, M. H. Roberts and R. Y. Moore, Effects of melatonin on 2-deoxy-[1-14 C]glucose uptake within rat suprachiasmatic nucleus. Am. J. Physiol. 255(2), R332-R337 (1988).

54. S. Shibata, V. M. Cassone and R. Y. Moore, Effects of melatonin on neuronal activity in the rat suprachiasmatic nucleus in vitro. Neurosci. Lett. 97, 140-144 (1989).

55. R. R. Margraf and G. R. Lynch, An in vitro circadian rhythm of melatonin sensitivity in the suprachiasmatic nucleus of the Djungarian hamster, Phodopus sungorus. Brain Res. 609, 45-50 (1993).

56. A. J. McArthur, M. U. Gillette and R. A. Prosser, Melatonin directly resets the rat suprachiasmatic circadian clock in vitro. Brain Res. 565, 158-161 (1991).

57. A. J. McArthur, A. E. Hunt and M. U. Gillette, Melatonin action and signal transduction in the rat suprachiasmatic circadian clock: activation of protein kinase $\mathrm{C}$ at dusk and dawn. Endocrinology 138(2), 627-634 (1997).

58. A. E. Hunt, W. M. Al-Ghoul, M. U. Gillette and M. L. Dubocovich, Activation of MT2 melatonin receptors in rat suprachiasmatic nucleus phase advances the circadian clock. Am. J. Physiol. 280, C110-C118 (2001).

59. T. Shirakawa and R. Y. Moore, Glutamate shifts the phase of the circadian neuronal firing rhythm in the rat suprachiasmatic nucleus in vitro. Neurosci. Lett. 178, 47-50 (1994).

60. J. M. Ding, D. Chen, E. T. Weber, L. E. Faiman, M. A. Rea and M. U. Gillette, Resetting the biological clock: mediation of nocturnal circadian shifts by glutamate and NO. Science 266, 1713-1717 (1994).

61. S. Shibata, A. Watanabe, T. Hamada, M. Ono and S. Watanabe, N-methyl-Daspartate induces phase shifts in circadian rhythm of neuronal activity of rat SCN in vitro. Am. J. Physiol. 267(2), R360-R364 (1994).

62. A. Watanabe, T. Hamada, S. Shibata and S. Watanabe, Effects of nitric oxide synthase inhibitors on N-methyl-D-aspartate-induced phase delay of circadian 
rhythm of neuronal activity in the rat suprachiasmatic nucleus in vitro. Brain Res. 646, 161-164 (1994).

63. A. Watanabe, M. Ono, S. Shibata and S. Watanabe, Effect of a nitric oxide synthase inhibitor, N-nitro-L-arginine methylester, on light-induced phase delay of circadian rhythm of wheel-running activity in golden hamsters. Neurosci. Lett. 192, 25-28 (1995).

64. E. T. Weber, R. L. Gannon, A. M. Michel, M. U. Gillette and M. A. Rea, Nitric oxide synthase inhibitor blocks light-induced phase shifts of the circadian activity rhythm, but not c-fos expression in the suprachiasmatic nucleus of the Syrian hamster. Brain Res. 692, 137-142 (1995).

65. J. M. Ding, G. F. Buchanan, S. A. Tischkau, et al., A neuronal ryanodine receptor mediates light-induced phase delays of the circadian clock. Nature 394, 381384 (1998).

66. J. M. Ding, L. E. Faiman, W. J. Hurst, L. R. Kuriashkina and M. U. Gillette, Resetting the biological clock: mediation of nocturnal CREB phosphorylation via light, glutamate, and nitric oxide. J. Neurosci. 17(2), 667-675 (1997).

67. S. A. Tischkau, J. W. Mitchell, S.-H. Tyan, G. F. Buchanan and M. U. Gillette, $\mathrm{Ca} 2+/ \mathrm{cAMP}$ response element-binding protein (CREB)-dependent activation of Per1 is required for light-induced signaling in the suprachiasmatic nucleus circadian clock. J. Biol. Chem. 278(2), 718-723 (2003).

68. P. C. Yannielli and M. E. Harrington, Neuropeptide Y applied in vitro can block the phase shifts induced by light in vivo. Neuroreport 11(7), 1587-1591 (2000).

69. E. M. Mintz, A. M. Jasnow, C. F. Gillespie, K. L. Huhman and H. E. Albers, GABA interacts with photic signaling in the suprachiasmatic nucleus to regulate circadian phase shifts. Neuroscience 109(4), 773-778 (2002).

70. R. A. Prosser, Glutamate blocks serotonergic phase advances of the mammalian circadian pacemaker through AMPA and NMDA receptors. J. Neurosci. 21(19), 7815-7822 (2001).

71. M. J. Bradbury, W. C. Dement and D. M. Edgar, Serotonin-containing fibers in the suprachiasmatic hypothalamus attenuate light-induced phase delays in mice. Brain Res. 768, 125-134 (1997).

72. A. L. Bergstrom, J. Hannibal, P. Hindersson and J. Fahrenkrug, Light-induced phase shift in the Syrian hamster (Mesocricetus auratus) is attenuated by the PACAP receptor antagonist PACAP6-38 or PACAP immunoneutralization. Eur. J. Neurosci. 18, 2552-2562 (2003).

73. D. Chen, G. F. Buchanan, J. M. Ding, J. Hannibal and M. U. Gillette, Pituitary adenylyl cyclase-activating peptide: a pivotal modulator of glutamatergic regulation of the suprachiasmatic circadian clock. Proc. Natl. Acad. Sci. U.S.A. 96, 13468-13473 (1999).

74. S. A. Tischkau, E. A. Gallman, G. F. Buchanan and M. U. Gillette, Differential cAMP gating of glutamatergic signaling regulates long-term state changes in the suprachiasmatic circadian clock. J. Neurosci. 20(20), 7830-7837 (2000).

75. M. Zatz and M. J. Brownstein, Intraventricular carbachol mimics the effects of light on the circadian rhythm in the rat pineal gland. Science 203, 358-360 (1979).

76. M. Zatz and M. A. Herkenham, Intraventricular carbachol mimics the phaseshifting effect of light on the circadian rhythm of wheel-running activity. Brain Res. 212, 234-238 (1981).

77. D. J. Earnest and F. W. Turek, Role for acetylcholine in mediating effects of light on reproduction. Science 219, 77-79 (1983). 
78. N. Murakami, K. Takahashi and K. Kawashima, Effect of light on the acetylcholine concentrations of the suprachiasmatic nucleus in the rat. Brain Res. 311, 358-360 (1984).

79. R. J. Wenthold. Glutamate and aspartate as neurotransmitters for the auditory nerve. In: Glutamate as a Neurotransmitter, edited by G. DiChiara and G. L. Gessa (Raven Press, New York, 1981) pp. 69-78.

80. O. Yasuhara, I. Tooyama, Y. Aimi, et al., Demonstration of choinergic ganglion cells in rat retina: expression of an alternative splice variant of choline acetyltransferase. J. Neurosci. 23(7), 2872-2881 (2003).

81. J. R. Pauly and N. D. Horseman, Anticholinergic agents do not block lightinduced circadian phase shifts. Brain Res. 348, 163-167 (1985).

82. C. S. Colwell, C. M. Kaufman and M. Menaker, Phase-shifting mechanisms in the mammalian circadian system: new light on the carbachol paradox. J. Neurosci. 13(4), 1454-1459 (1993).

83. C. Liu and M. U. Gillette, Cholinergic regulation of the suprachiasmatic nucleus circadian rhythm via a muscarinic mechanism at night. J. Neurosci. 16(2), 744-751 (1996).

84. G. F. Buchanan and M. U. Gillette. Carbachol directly stimulating the SCN induces phase advances in mouse circadian rhythms throughout the night in vitro and in vivo. Abstract presented at: Society for Neuroscience, 2001.

85. G. F. Buchanan, L. R. Artinian, S. E. Hamilton, N. M. Nathanson and M. U. Gillette. The M1 muscarinic acetylcholine receptor is a necessary component in cholinergic circadian signaling. Abstract presented at: Society for Neuroscience, 2000.

86. G. F. Buchanan. Thesis: Cholinergic regulation of the mammalian circadian system: analysis of cholinergic-induced phase shifting in vivo and in vitro in wildtype and M1 knockout mice: Molecular and Integrative Physiology, University of Illinois at Urbana-Champaign, 2002.

87. A. A. Borbely, A two process model of sleep regulation. Hum. Neurobiol. 1(3), 195-204 (1982).

88. A. A. Borbely, Processes underlying sleep regulation. Horm. Res. 49, 114-117 (1998).

89. N. Ibuka and H. Kawamura, Loss of circadian rhythm in sleep-wakefulness cycle in the rat by suprachiasmatic nucleus lesions. Brain Res. 96, 76-81 (1975).

90. N. Ibuka, S.-I. T. Inouye and H. Kawamura, Analysis of sleep-wakefulness rhythms in male rats after suprachiasmatic nucleus lesions and ocular enucleation. Brain Res. 122, 33-47 (1977).

91. W. B. Mendelson, B. M. Bergmann and A. Tung, Baseline and post-deprivation recovery sleep in SCN-lesioned rats. Brain Research 980, 185-190 (2003).

92. I. Tobler, A. A. Borbely and G. Groos, The effect of sleep deprivation on sleep in rats with suprachiasmatic lesions. Neurosci. Lett. 42, 49-54 (1983).

93. R. E. Mistlberger, B. M. Bergmann, W. Waldenar and A. Rechtschaffen, Recovery sleep following sleep deprivation in intact and suprachiasmatic nuclei lesioned rats. Sleep 6(3), 217-233 (1983).

94. D. M. Edgar, W. C. Dement and C. A. Fuller, Effect of SCN lesions on sleep in squirrel monkeys: evidence for opponent processes in sleep-wake regulation. J. Neurosci. 13(3), 1065-1079 (1993).

95. M. C. Antle and R. E. Mistlberger, Circadian clock resetting by sleep deprivation without exercise in the Syrian hamster. J. Neurosci. 20(24), 9326-9332 (2000). 
96. E. Challet, F. W. Turek, M.-A. Laute and O. V. Reeth, Sleep deprivation decreases phase-shift responses of circadian rhythms to light in the mouse: role of serotonergic and metabolic signals. Brain Res. 909, 81-91 (2001).

97. S. F. Glotzbach, C. M. Cornett and H. C. Heller, Activity of suprachiasmatic and hypothalamic neurons during sleep and wakefulness in the rat. Brain Res. 419, 279-286 (1987).

98. T. Deboer, M. J. Vansteensel, L. Detari and J. H. Meijer, Sleep states alter activity of suprachiasmatic nucleus neurons. Nat. Neurosci. 6(10), 1086-1090 (2003). 